\title{
Targeting KPNB1 overcomes TRAIL resistance by regulating DR5, Mcl-1 and FLIP in glioblastoma cells
}

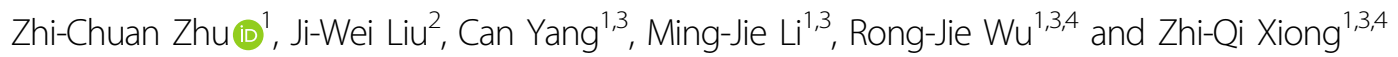

\begin{abstract}
Tumor necrosis factor-related apoptosis-inducing ligand (TRAIL) is a cytokine with potential anticancer effect, but innate and adaptive TRAIL resistance in majority of cancers limit its clinical application. Karyopherin $\beta 1$ (KPNB1) inhibition in cancer cells has been reported to abrogate the nuclear import of TRAIL receptor DR5 and facilitate its localization on the cell surface ready for TRAIL stimulation. However, our study reveals a more complicated mechanism. Genetic or pharmacological inhibition of KPNB1 potentiated TRAlL-induced apoptosis selectively in glioblastoma cells mainly by unfolded protein response (UPR). First, it augmented ATF4-mediated DR5 expression and promoted the assembly of death-inducing signaling complex (DISC). Second, it freed Bax and Bak from Mcl-1. Third, it downregulated FLIPL and FLIP , inhibitors of caspase-8 cleavage, partly through upregulating ATF4-induced 4E-BP1 expression and disrupting the cap-dependent translation initiation. Meanwhile, KPNB1 inhibition-induced undesirable autophagy and accelerated cleaved caspase-8 clearance. Inhibition of autophagic flux maintained cleaved caspase-8 and aggravated apoptosis induced by KPNB1 inhibitor plus TRAIL, which were abolished by caspase-8 inhibitor. These results unveil new molecular mechanism for optimizing TRAlL-directed therapeutic efficacy against cancer.
\end{abstract}

\section{Introduction}

Tumor necrosis factor-related apoptosis-inducing ligand (TRAIL) belongs to the tumor necrosis factor superfamily of cytokines and is involved in inflammation and immunosurveillance. It is expressed in both normal and tumor cells. TRAIL induces apoptosis by engaging its functional receptors DR4 (TRAIL-R1) and DR5 (TRAILR2). Upon TRAIL stimulation, TRAIL receptors undergo homotrimerization and recruit Fas-associated protein with death domain (FADD). FADD turns to recruit caspase-8. Assembly of this death-inducing signaling complex (DISC) promotes caspase-8 processing and

\footnotetext{
Correspondence: Zhi-Qi Xiong (xiongzhiqi@ion.ac.cn)

${ }^{1}$ State Key Laboratory of Neuroscience, Institute of Neuroscience, CAS Center for Excellence in Brain Science and Intelligence Technology, Chinese Academy of Sciences, Shanghai, China

${ }^{2}$ Shanghai Mental Health Center, Shanghai Jiao Tong University School of Medicine, Shanghai, China

Full list of author information is available at the end of the article.

Edited by M. Agostini
}

activation. In certain types of cells, cleaved caspase- 8 directly cleaves effector caspases like caspase- 3 to induce apoptosis, while in other cells the intrinsic mitochondrial apoptotic signaling amplifies the death signal. In the latter case, Bid, truncated by cleaved caspase- 8 , translocates to the mitochondria and binds pro-survival $\mathrm{Bcl}-2$ proteins like Bcl-xL or pro-apoptotic Bcl-2 proteins like Bax and Bak to facilitate mitochondria outer membrane permeabilization (MOMP). This leads to the release of cytochrome $\mathrm{c}$ and other pro-apoptotic factors into the cytosol, the activation of effector caspases and the induction of apoptosis $^{1,2}$.

Clinical trials revealed the safety but disappointed clinical benefits of TRAIL-based therapies ${ }^{2,3}$. Multiple factors in TRAIL receptor signaling determine TRAIL responsiveness, including the expression, localization, and clustering of TRAIL receptors, the assembly and distribution of DISC and the expression of $\mathrm{Bcl}-2$ family proteins and inhibitors of apoptosis proteins ${ }^{1,4}$.

\section{(c) The Author(s) 2019}

(c) Open Access This article is licensed under a Creative Commons Attribution 4.0 International License, which permits use, sharing, adaptation, distribution and reproduction cc) in any medium or format, as long as you give appropriate credit to the original author(s) and the source, provide a link to the Creative Commons license, and indicate if changes were made. The images or other third party material in this article are included in the article's Creative Commons license, unless indicated otherwise in a credit line to the material. If material is not included in the article's Creative Commons license and your intended use is not permitted by statutory regulation or exceeds the permitted use, you will need to obtain permission directly from the copyright holder. To view a copy of this license, visit http://creativecommons.org/licenses/by/4.0/. 
Therapeutic strategies modulating these factors to improve TRAIL response are urgently needed.

Karyopherin $\beta 1$ (KPNB1) participates in the nuclear import of many cancer-associated proteins including DR $5^{5-8}$. KPNB1 transports DR5 into the nucleus, while knocking down KPNB1 restores DR5 protein level on the cell surface and TRAIL sensitivity of cancer cells ${ }^{8}$. We demonstrated previously that KPNB1 inhibition perturbed proteostasis and activated PERK signaling branch of unfolded protein response (UPR) in glioblastoma cells ${ }^{9}$. Given that PERK branch regulates the expression of DR5 and other determinants of TRAIL susceptibility ${ }^{10,11}$, we envisage that KPNB1 inhibition may overcome TRAIL resistance via UPR rather than simply abolishing DR5 nuclear import. In the present study, we show that KPNB1 inhibition results in DR5 upregulation, Mcl-1 disability and FLIP downregulation via UPR. Combination of KPNB1 inhibitor and TRAIL along with the lysosome inhibitor uncoupling pro-survival autophagy has potential in cancer treatment.

\section{Results}

Inhibition of KPNB1 sensitizes glioblastoma cells to TRAILinduced apoptosis

It was reported that KPNB1 knockdown primed cancer cells to TRAIL-induced apoptosis by upregulating cell surface DR $5^{8}$. Consistently, in our study, KPNB1 shRNAs (shKPNB1-1, 2) or specific inhibitor importazole (IPZ) potentiated TRAIL cytotoxicity in A172, U87, U118, U251 human glioblastoma cells but not in human fetal astrocytes (HA) (Fig. 1a-c). In A172 and U87 cells, KPNB1 inhibition plus TRAIL-induced robust cell death and activation of the death receptor apoptotic signaling in terms of the cleavage of caspase- 8 (p43/p41), Bid, caspase3 (intermediate p19 and effector p17/p12) and PARP (Fig. 1d-g). Such effects were weaker in U251, U118 cells (Fig. 1d, e) and were weakest in HA cells (Fig. 1d-g). These results suggest that KPNB1 inhibition synergizes with TRAIL to selectively induce apoptosis in glioblastoma cells.

\section{KPNB1 inhibition increases total and cell surface DR5 level} in glioblastoma cells

In consistent with previous findings ${ }^{8}$, both KPNB1 knockdown and IPZ treatment increased cell surface DR5 levels in U87 cells (Fig. 2a-d). Besides, KPNB1 knockdown attenuated DR5 nuclear import in U87 cells (Fig. 2e). Inconsistently, however, KPNB1 inhibition also increased total DR5 levels in U87 and U251 cells in a time- and dose-dependent manner (Fig. 2f-h). Meanwhile, KPNB1 knockdown and IPZ treatment did not generally increase cell surface DR4 level in U87 and U251 cells (Fig. 2i, j). DR4 mean fluorescence intensity (MFI) was comparable to that of isotype IgG in U87 and U251 cells and lower than DR5 MFI in U87 cells (Fig. 2a-d, i, j), recapitulating previous result ${ }^{12}$ and indicating low cell surface expression of DR4 in these cells. KPNB1 knockdown upregulated total DR4 and DR5 in all tested glioblastoma cell lines (Fig. 2k). Each KPNB1 inhibitor (IPZ, IVM, and INI-43) upregulated DR5 in U87, U118, and U251 cells. However, IPZ upregulated DR4 only in U118 cells and INI-43 upregulated DR4 only in U87 and U118 cells (Fig. 2l). U118 cells also had high DR5 and low DR4 on the cell surface despite expressing relatively high total DR4 and low total DR5 (Fig. 2k, l and Supplementary Fig. S1A, B). IPZ but not KPNB1 knockdown increased cell surface DR4 and DR5 in U118 cells (Supplementary Fig. S1A, B), possibly explaining lower TRAIL response in shKPNB1 cells than that in IPZ-treated cells (Fig. 1d, e). These results suggest that KPNB1 inhibition upregulates cell surface DR5 level by inducing DR5 expression in addition to suppressing DR5 internalization, while it does not uniformly increase cell surface DR4 level albeit affecting total DR4 level in some cases.

\section{KPNB1 inhibition enhances TRAIL sensitivity by promoting ATF4-mediated DR5 expression}

In U87 cells, IPZ dose-dependent DR5 upregulation correlates with the synergism of IPZ and TRAIL (Fig. 1c, e and g). KPNB1 knockdown or IPZ treatment strongly promoted TRAIL-induced assembly of DISC core members caspase-8, FADD, and DR5 but not DR4 (Fig. 3a, b). Apoptosis induced by KPNB1 inhibition and TRAIL treatment was reversed by DR5 knockdown in U87 cells (Fig. 3c, d). Antagonistic antibody to DR5 neutralizing cell surface DR $5^{13}$ also reversed such apoptosis in U87 cells, whereas the effect of antagonistic antibody to cell surface DR4 was weaker (Fig. 3e, f). Similar result was obtained in IPZ-treated U118 cells with upregulated cell surface DR4 and DR5 (Supplementary Fig. S1C). These results suggest that KPNB1 inhibition-upregulated cell surface DR5 mainly amplifies the death receptor signaling transduction favoring TRAIL-triggered apoptosis in tested glioblastoma cells.

ER stress regulators, p53, and NF- $\mathrm{kB}$ regulate DR5 transcription ${ }^{10,14}$. KPNB1 inhibition activated PERK signaling branch of UPR in U87 cells (Fig. 3g). Knocking down ATF4 reversed the upregulation of mRNA, total protein and cell surface DR5 upon KPNB1 inhibition (Fig. 2a-d and Fig. 3h-k). Knocking down CHOP that commonly enhances DR5 transcription under ER stress ${ }^{15,16}$ had a weaker effect (Supplementary Fig. S2AD). KPNB1 inhibition also slowed DR5 degradation and downregulated E3 ligase c-Cbl which accounts for DR5 degradation and early phase TRAIL resistance ${ }^{17}$ (Supplementary Fig. S3A, B). Overexpression of c-Cbl reversed DR5 level mildly that was insufficient to attenuate apoptosis upon KPNB1 inhibition and TRAIL stimulation 


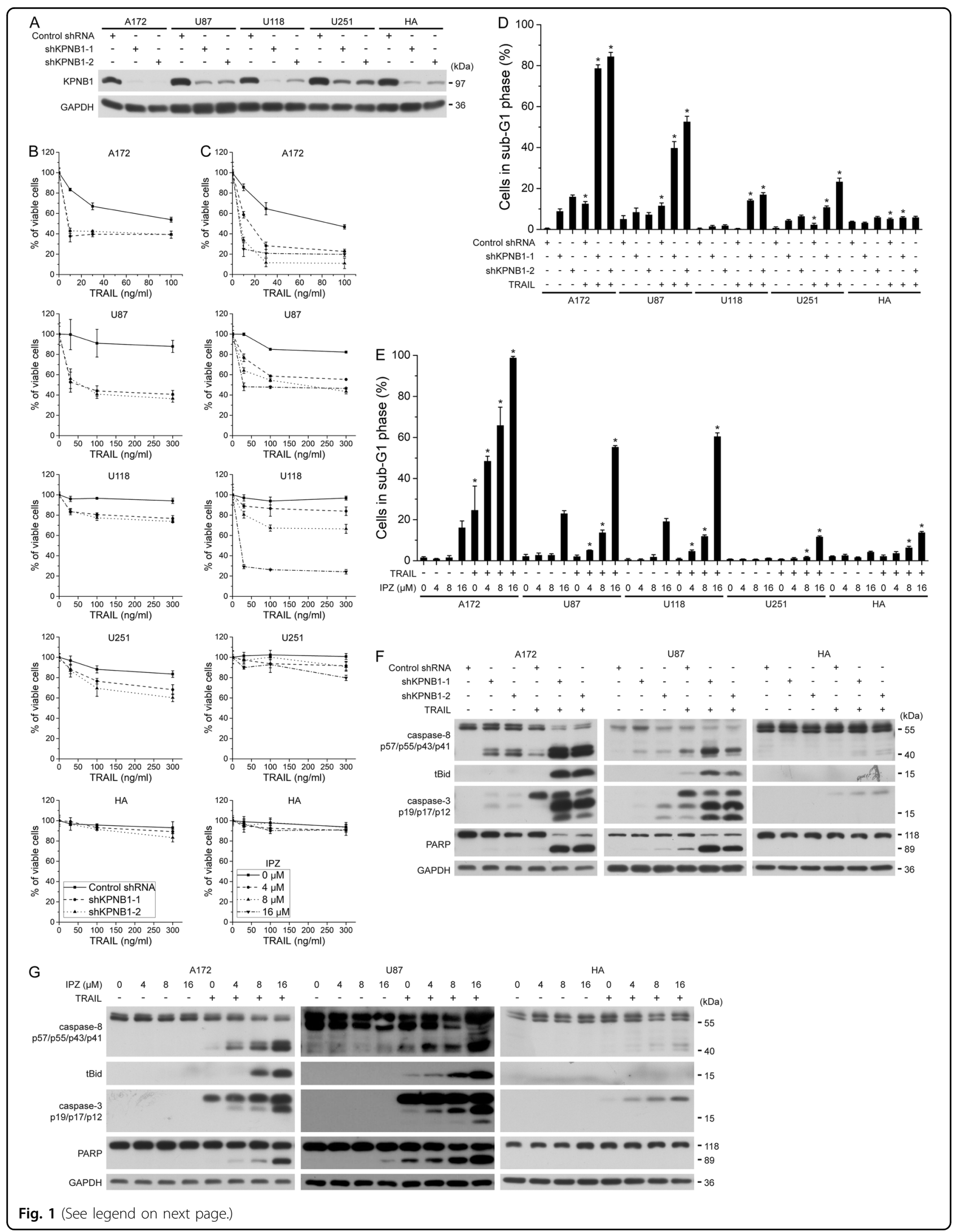


(see figure on previous page)

Fig. 1 Inhibition of KPNB1 sensitizes glioblastoma cells to TRAIL-induced apoptosis. a A172, U87, U118, U251, and HA cells were infected lentiviruses encoding shKPNB1s and a scrambled shRNA (Control shRNA). Knockdown efficacy of shRNAs was validated by western blot. Molecular weight of proteins is indicated at the right-hand side. b, c Cells either expressing shKPNB1s (b) or pretreated with indicated dose of IPZ for $24 \mathrm{~h}$ (c) were treated with indicated dose of human recombinant TRAlL for $24 \mathrm{~h}$. Cell viability was measured by MTT assay. Results represent the mean \pm SD from one of the three independent experiments in triplicates. d, e Cells pretreated as indicated were treated with TRAIL ( $30 \mathrm{ng} / \mathrm{ml})$ for $24 \mathrm{~h}$. The percentage of apoptotic cells was analyzed by flow cytometry. Results represent mean \pm SD from three independent experiments. ${ }^{*} P<0.05$ compared with the corresponding group without TRAIL treatment. f, $\mathbf{g}$ U87 cells pretreated as indicated were further treated with TRAIL (30 ng/ml) for $6 \mathrm{~h}$. Proteins in the death receptor signaling were analyzed by western blot. GAPDH was used as the loading control

(Supplementary Fig. S3C, D). These results suggest that KPNB1 inhibition-upregulated DR5, mainly through ATF4, enhances TRAIL sensitivity in glioblastoma cells.

\section{KPNB1 inhibition compromises Mcl-1 to enhance TRAIL sensitivity}

Mcl-1 prevents MOMP when TRAIL-induced death receptor signaling turns to activate the mitochondrial apoptotic signaling ${ }^{2}$. In U87 cells, KPNB1 knockdown or IPZ treatment decreased binding of Mcl-1 to Bax and Bak (Fig. 4a, b). KPNB1 inhibition-upregulated Noxa, a transcriptional target of ATF4 and a Mcl-1 antagonizer ${ }^{18}$, and its binding to Mcl-1 (Fig. 4a, b). However, Noxa knockdown did not rescue apoptosis induced by IPZ and TRAIL (Supplementary Fig. S4). Overexpression of the nondegradable Mcl-1 (T92A) mutant partly reversed KPNB1 inhibition and TRAIL-induced apoptosis in U87 cells (Fig. 4c, d). These results suggest that KPNB1 inhibition compromises the anti-apoptotic function of Mcl-1 to foster TRAIL-induced apoptosis.

\section{KPNB1 inhibition cripples FLIP translation and TRAIL tolerance by ATF4/4E-BP1 axis}

UPR halts massive mRNA translation to relieve ER protein overload $^{19}$, which may impair the expression of short-lived proteins. The short-lived protein FLIP inhibits caspase- 8 processing and activation and causes TRAIL resistance. FLIP isoform FLIP $_{L}$ played more important role than FLIP in preventing U87 cells from TRAILinduced apoptosis (Supplementary Fig. S5A, B). KPNB1 knockdown or IPZ treatment downregulated both FLIP $_{\mathrm{L}}$ and FLIP in U87 cells. Overexpression of FLIP $_{L}$ greatly while FLIP $_{S}$ moderately reversed TRAIL-induced apoptosis in these cells (Fig. 5a, b), indicating KPNB1 inhibition downregulates FLIP to increase TRAIL sensitivity. KPNB1 inhibition decreased neither mRNA expression nor protein stability of FLIP (Supplementary Fig. S5C, D). Therefore, factors other than transcription, mRNA processing and protein turnover regulated FLIP expression. During the treatment of actinomycin D (Act D) and MG132 that inhibit transcription and protein degradation respectively, protein levels of $\mathrm{FLIP}_{\mathrm{L}}$ and $\mathrm{FLIP}_{\mathrm{S}}$ in control cells increased more than in KPNB1-inhibited U87 cells (Supplementary Fig. S5E), suggesting the change of FLIP protein synthesis. To verify the translational regulation of FLIP, we investigated the cap-dependent translation initiation signaling, in which 4E-BP1 and eIF2 $\alpha$ are controlled by UPR to suppress cap-dependent translation initiation $^{19-22}$. In addition to the upregulated p-eIF2 $\alpha$, KPNB1 knockdown or IPZ treatment in U87 cells upregulated $\alpha$ isoform of $4 \mathrm{E}-\mathrm{BP} 1(4 \mathrm{E}-\mathrm{BP} 1 \alpha)$ that competed with eIF4G for binding $\mathrm{m}^{7} \mathrm{GTP}$ (mimicking mRNA $5^{\prime}$ cap)-bound eIF4E (Fig. 5c), thus impairing the integrity of cap-dependent translation initiation complex eIF4F. Therefore, IPZ treatment decreased total protein synthesis in U87 and U251 (Fig. 5d). Nevertheless, mTORregulated p-4E-BP1 (T37/46) which influences 4E-BP1 activity was not altered (Fig. 5c). 4E-BP1 knockdown reconstituted cap-bound eIF4E/eIF4G complex (Fig. 5e, f), restored FLIP levels (Fig. 5g, h) and nullified TRAILinduced apoptosis (Fig. 5i, j) upon KPNB1 inhibition in U87 cells. Unexpectedly, 4E-BP1 knockdown suppressed FLIP $_{\mathrm{L}}$ expression (Fig. 5g, h). Considering 4E-BP1 does not regulate FLIP $_{\mathrm{L}}$ translation in glioblastoma cells ${ }^{23}$, we no longer investigated their relationship in the following study. ATF4-mediated KPNB1 inhibition-induced upregulation of $4 \mathrm{E}-\mathrm{BP} 1$ transcript and $4 \mathrm{E}-\mathrm{BP} 1 \alpha$ protein (Fig. 5k, l), recapitulating the previous finding that ATF4 induced 4E-BP1 under ER stress ${ }^{21}$. However, ATF4 knockdown failed to rescue shKPNB1/TRAIL-induced apoptosis (Supplementary Fig. S6). EIF2 $\alpha$ (S52A) overexpression suppressed IPZ-induced upregulation of ATF4 but not 4E-BP1 $\alpha$ (Fig. $5 \mathrm{~g}$ ), possibly due to other unknown signalings regulated by the functional nonphosphorylated eIF2 $\alpha$. Moreover, bioinformatics analysis indicated that KPNB1 mRNA expression had moderate negative correlation with that of ATF4 in glioblastoma multiforme but not low grade glioma (Supplementary Fig. S7). These results suggest that KPNB1 inhibition upregulates $4 \mathrm{E}-\mathrm{BP} 1$ by ATF4, which reduces the capdependent translation of FLIP $_{S}$ and TRAIL resistance.

\section{KPNB1 inhibition-induced autophagy targets cleaved caspase-8 for clearance and counteracts apoptosis}

KPNB1 knockdown or IPZ treatment activated autophagy, as revealed by the diffuse bands of the lysosome marker LAMP1 and upregulation of autophagy markers LC3B-II and/or p62 (Fig. 6a). Bioinformatics analysis 


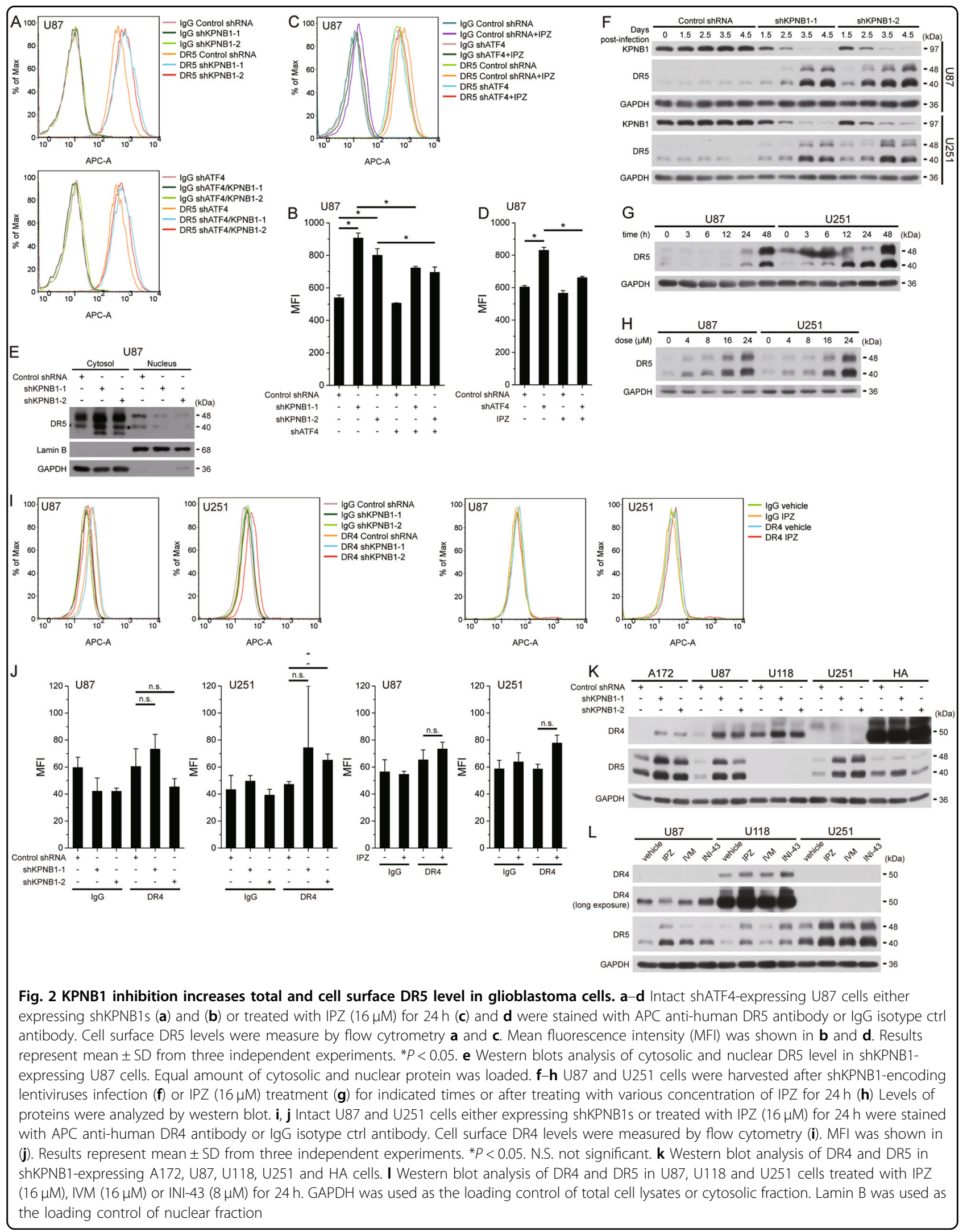




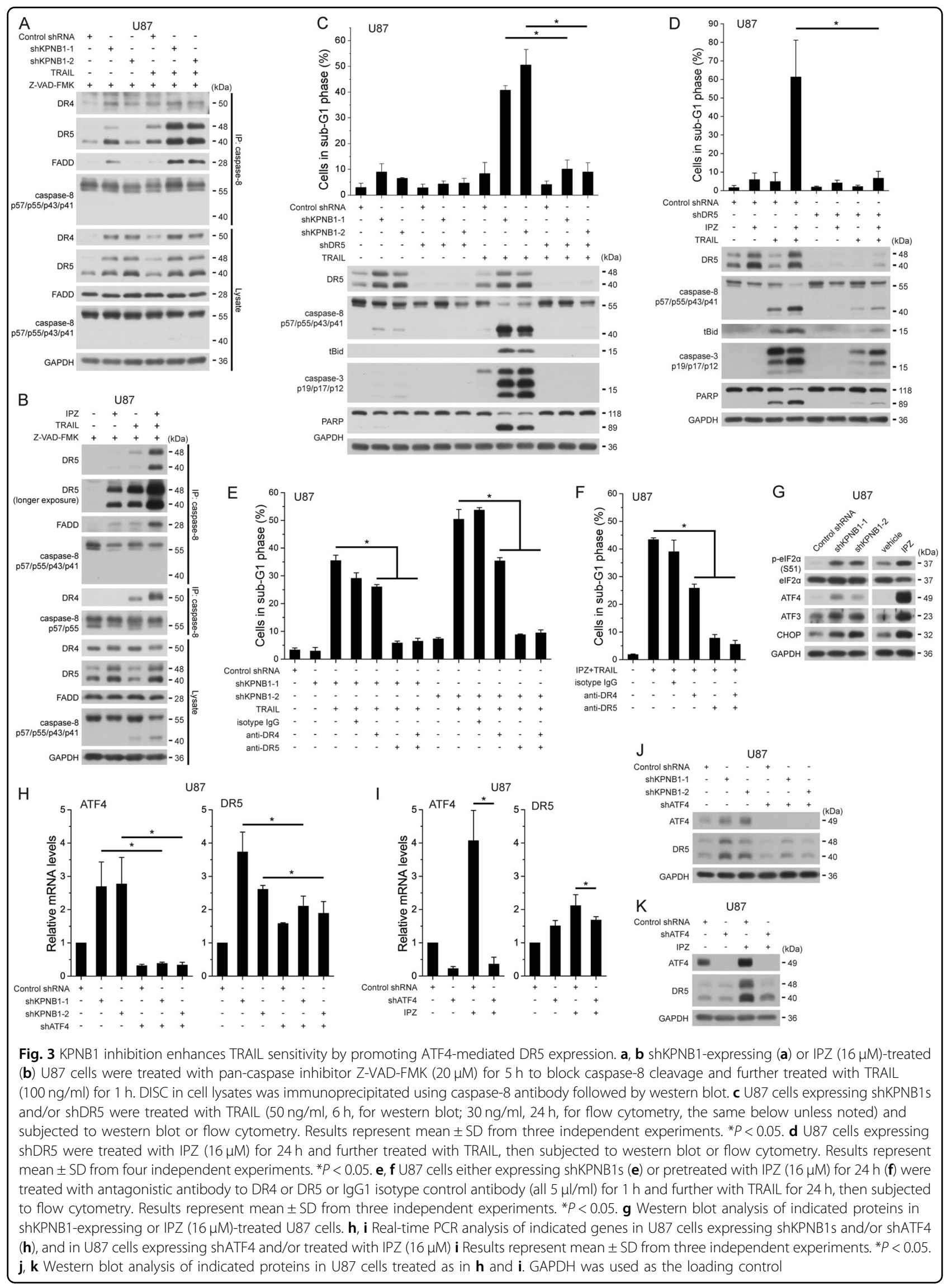




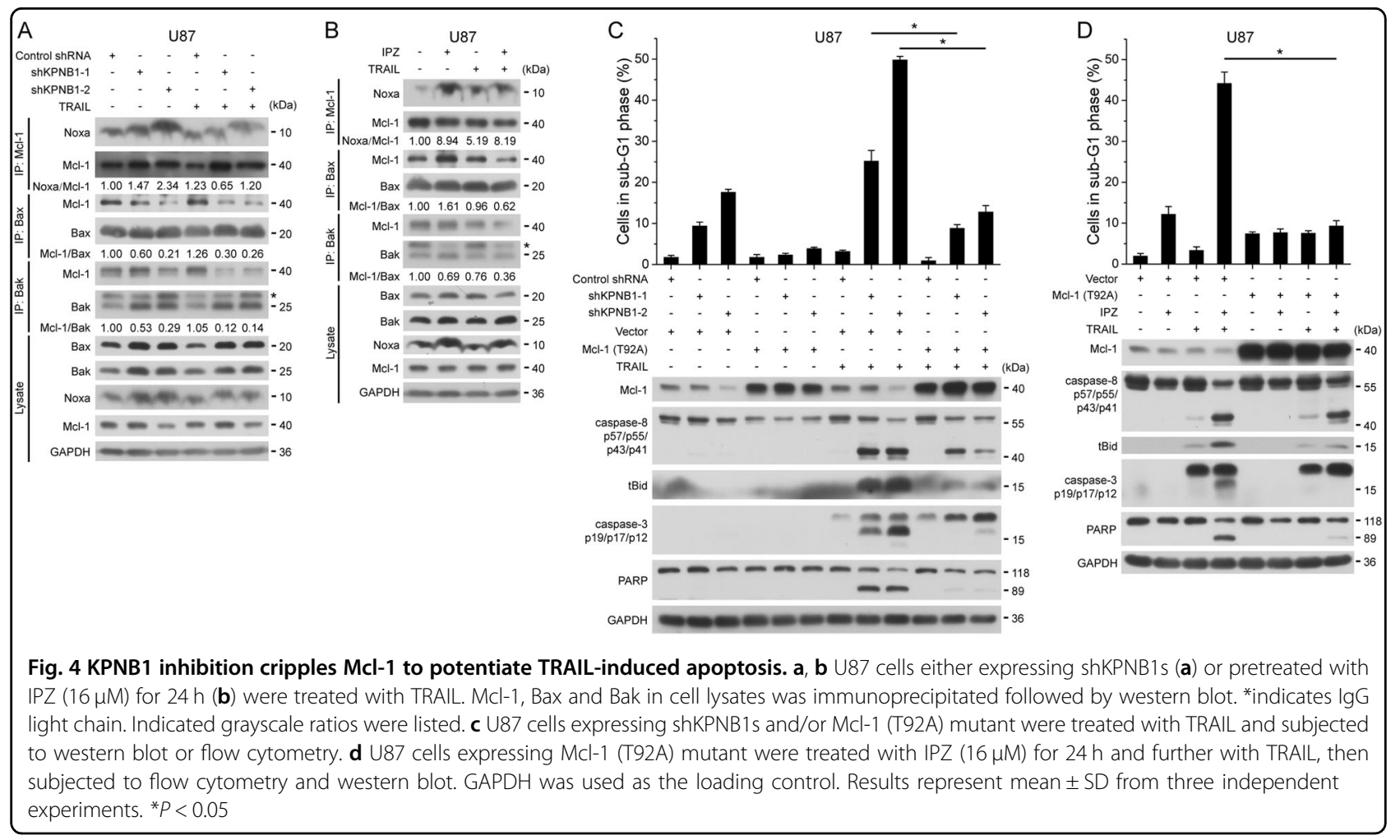

supported a negative correlation between mRNA expression of KPNB1 and LC3B in glioblastoma multiforme (Supplementary Fig. S7). LC3B-II level that inversely correlates with autophagosome clearance was higher in U87 cells than in U251 cells with or without KPNB1 inhibition (Fig. 6a). Given that U251 cells were less sensitive to KPNB1 inhibition/TRAIL combination than U87 cells (Fig. 1b-e), we predicted that autophagic flux level somehow negatively correlated with the efficacy of such combination. We used IPZ and another KPNB1 inhibitor ivermectin (IVM), which has similar effects on UPR, protein synthesis and downstream molecules linked to TRAIL sensitivity as IPZ (Supplementary Fig. S8A and Fig. 5d) in the remainder study. Knocking down key autophagy regulators ATG5, p62, and LC3B all further enhanced apoptosis in U251 and U87 cells following KPNB1 inhibitor/TRAIL treatment (Fig. 6b, c and Supplementary Fig. S8B, C). Lysosome inhibitors chloroquine (CQ) or bafilomycin A1 (Baf-A1) had similar effect on apoptosis induction (Fig. 6d, e and Supplementary Fig. S8D-F). These results substantiate a pro-survival role of autophagy in KPNB1 inhibitor/TRAIL treatment. In U87 and U251 cells, both knockdown of ATG5, p62 or $\mathrm{LC} 3 \mathrm{~B}$ and treatment of $\mathrm{CQ}$ or Baf-A1 upregulated the intermediate product $\mathrm{p} 43 / \mathrm{p} 41$ and the active product $\mathrm{p} 18$ of cleaved caspase-8, which facilitated the cleavage of Bid, caspase-3 and PARP (Fig. 6c, e and Supplementary Fig. S8C, E). Among them, p62 knockdown had the least effect on cleaved caspase- 8 accumulation and apoptosis promotion (Fig. 6b, c and Supplementary Fig. S8B, C), possibly because procaspase- 8 requires p62 for aggregation and cleavage upon TRAIL stimulation ${ }^{24}$. After cleavage caspase- 8 p43/p41 and p18 products are ubiquitinated for degradation and colocalized with the autophagolysosome probably for clearance ${ }^{25}$. KPNB1 inhibitor/TRAIL combination-induced p62 binding to p43/p41 and p18 in U87 and U251 cells and colocalization of p62, p43/p41/p18 and LC3 in U251 cells (Fig. 7a, b). Consistently, blocking lysosomal degradation with lysosome inhibitor or LC3B knockdown stabilized caspase-8 p43/p41 and p18 (Fig. 7c). Caspase-8 inhibitor Z-IETDFMK reversed cell apoptosis induced by KPNB1 inhibitor, TRAIL and autophagy inhibition (Fig. 7d, e and Supplementary Fig. S8H-J). Thus, blocking autophagy-lysosomal degradation prevents cleaved caspase- 8 from clearance and markedly augmented the synergism of KPNB1 inhibitor/TRAIL combination in glioblastoma cells.

\section{Combination of IVM, CQ, and TRAIL reduces tumor growth in vivo}

At last, we analyzed the anticancer activity of combination of KPNB1 inhibitor, lysosome inhibitor, and TRAIL in nude mice subcutaneously inoculated U87 cells. We purified sufficient amount of human recombinant soluble TRAIL, which is trimeric in PBS solution (Supplementary Fig. S9A, B). IVM with proved clinical safety was used as KPNB1 inhibitor for the therapy. IVM, CQ plus TRAIL better inhibited tumor growth than vehicle, 


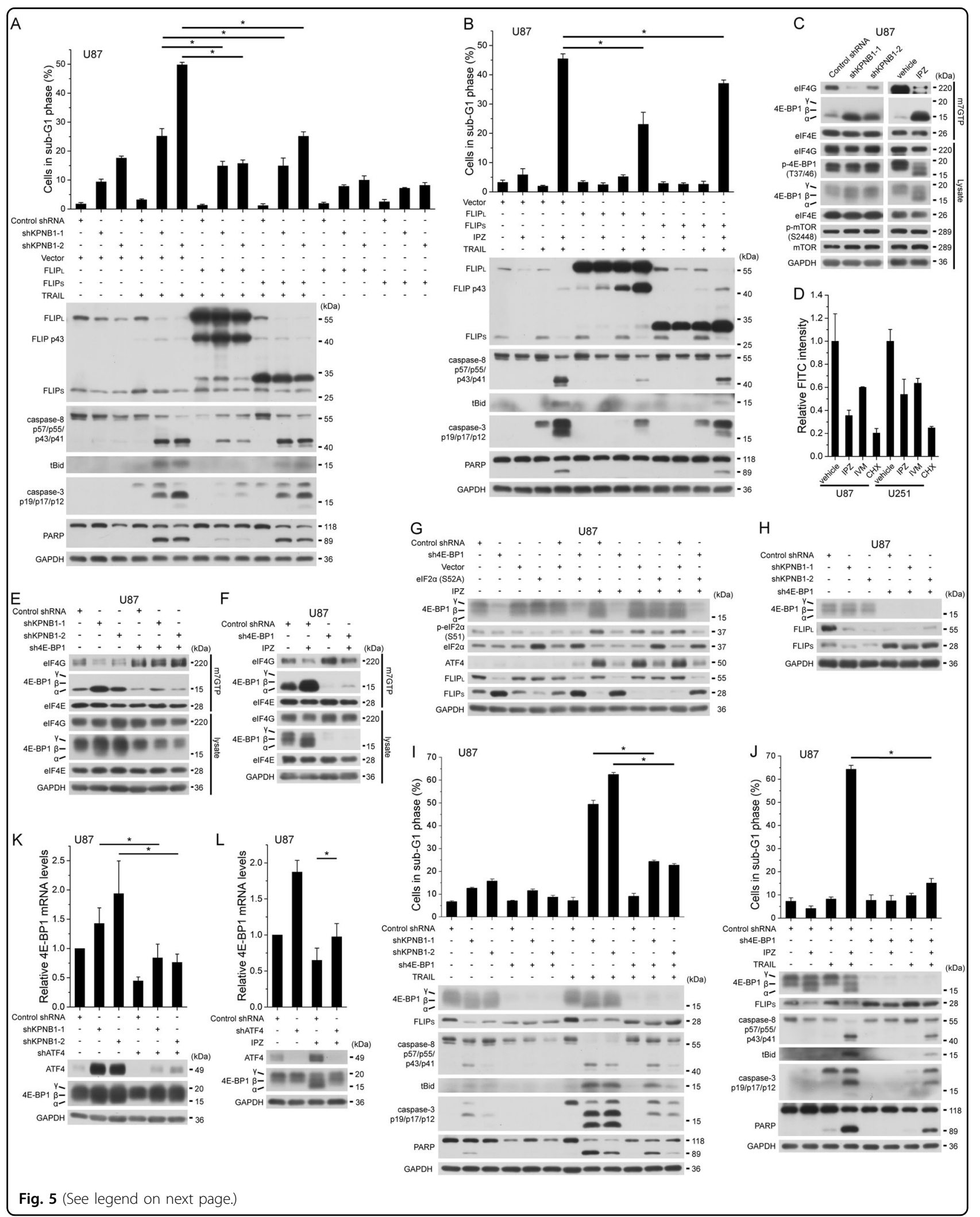


(see figure on previous page)

Fig. 5 KPNB1 inhibition cripples cap-dependent translation of FLIP and TRAIL tolerance by ATF4/4E-BP1 axis. a U87 cells expressing shKPNB1s and FLIP or FLIP were treated with TRAIL and subjected to western blot or flow cytometry. Results represent mean \pm SD from three

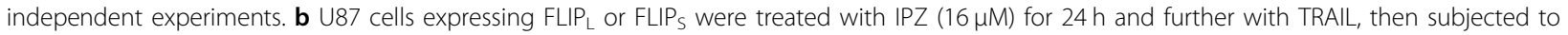
western blot and flow cytometry. Results represent mean \pm SD from three independent experiments. $\mathbf{c} U 87$ cells expressing shKPNB1s or treated with IPZ $(16 \mu \mathrm{M})$ for $24 \mathrm{~h}$ were subjected to cap-binding assay. $\mathbf{d}$ Protein synthesis was measured by OPP incorporation after treating U87 and U251 cells with $16 \mu \mathrm{M}$ IPZ or IVM for $24 \mathrm{~h}$, or with $100 \mu \mathrm{g} / \mathrm{ml} \mathrm{CHX}$ for $4 \mathrm{~h}$ as a negative control for protein synthesis Results represent mean \pm SD from one of the three independent experiments in triplicates. e U87 cells expressing shKPNB1s and/or sh4E-BP1 were subjected to cap-binding assay. $\mathbf{f}$ U87 cells expressing sh4E-BP1 were treated with IPZ (16 $\mu \mathrm{M})$ for $24 \mathrm{~h}$ and subjected to cap-binding assay. $\mathbf{g}$ U87 cells expressing sh4E-BP1 and/or elF2a (S52A) were treated with IPZ (16 $\mu \mathrm{M})$ for $24 \mathrm{~h}$ and subjected to western blot. $\mathbf{h}$ U87 cells from e were subjected to western blot. $\mathbf{i} \cup 87$ cells expressing sh4EBP1 were treated as in $\mathbf{a}$ and subjected to western blot and flow cytometry. Results represent mean \pm SD from three independent experiments. $\mathbf{j} \cup 87$ cells expressing shKPNB1s and/or sh4E-BP1 were treated as in $\mathbf{b}$ and subjected to western blot or flow cytometry. Results represent mean \pm SD from three independent experiments. $\mathbf{k}$ U87 cells expressing shKPNB1s and/or shATF4 were subjected to real-time PCR and western blot. Results represent mean \pm SD from three independent experiments. I U87 cells expressing shATF4 were treated with IPZ $(16 \mu \mathrm{M})$ for $24 \mathrm{~h}$ and subjected to real-time PCR and western blot. Results represent mean \pm SD from three independent experiments. GAPDH was used as the loading control. ${ }^{*} P<0.05$

single-treatment or double-treatment in early stage (day 1-5) but not in later stage (day 5-9) according to gradients of log-transformed tumor volume curves (Fig. 8a). The mean tumor volume of IVM + CQ + TRAIL group is $53 \%$ of that of vehicle group (Fig. 8b). Combination of IVM, CQ, and TRAIL was tolerated, with moderate weight loss late in the treatment (Fig. 8c). IVM and/or CQ enhanced TRAIL-induced cleavage of caspase- 8 and caspase- 3 in tumors (Fig. 8d, e). These results suggest that combination of IVM, CQ, and TRAIL reduces xenograft growth by inducing apoptosis. Nevertheless, TRAIL dosage $(100 \mu \mathrm{g})$ in this therapy was too low to synergize with IVM and/or CQ to reduce tumor growth and activate death receptor signaling (in terms of procaspase-8/ cleaved caspase- 8 ratio) as potent as those in vitro, especially when tumors were big. Raising TRAIL dose or replacing it with modified TRAIL to improve its activity or pharmacokinetic profile ${ }^{26,27}$ may solve this problem.

\section{Discussion}

In the present study, we delineate the molecular basis of KPNB1 inhibition in modulating TRAIL vulnerability of glioblastoma cells. Consistent with previous study, chemical or genetic inhibition of KPNB1 primes cells to TRAIL-induced apoptosis. However, rather than simply upregulating the cell surface DR5, KPNB1 inhibition triggers UPR to rewire the TRAIL receptor signaling and abrogate TRAIL resistance (Fig. 8f).

Earlier we found that KPNB1 inhibition in glioblastoma cells perturbed proteostasis and consequently activated UPR to alleviate stressful condition and to induce apoptosis of cells under chronic stress ${ }^{9}$. Therapies that activate UPR can sensitize cancer cells but not non-neoplastic cells to TRAIL treatment mainly through the following mechanisms. First, it upregulates DR5 expression commonly through the transcriptional factor CHOP and sometimes through ATF4 or IRE1 $\alpha-\mathrm{XBP} 1 \mathrm{~s}$ axis $^{28-30}$. Sometimes increased DR4 expression dominates apoptosis by TRAIL and UPR ${ }^{29,31}$. Second, it upregulates pro- apoptotic Bcl-2 proteins like Bim and Puma, which block the anti-apoptotic $\mathrm{Bcl}-2$ proteins ${ }^{32}$ to facilitate MOMP. Third, it inhibits translation initiation to downregulate proteins like Mcl-1, which block the death receptor signaling ${ }^{33-35}$. Although the expression of TRAIL receptors does not correlate with TRAIL sensitivity of cancer cells in general $^{1,4}$, ample evidence supports that upregulating TRAIL receptors overcomes TRAIL resistance ${ }^{15,16,32}$. Like many UPR sensitizers, KPNB1 inhibition enhances ATF4dependent DR5 transcription. DR5 upregulation is less dependent on CHOP because KPNB1 inhibition causes cytosolic retention of UPR-upregulated $\mathrm{CHOP}^{9}$. KPNB1 inhibition stabilizes DR5 by mechanisms beside c-Cbl downregulation. As a result, DR5 upregulation increases cell surface DR5 level, DISC formation and apoptotic signaling transduction upon KPNB1 inhibition and TRAIL treatment. On the other hand, KPNB1 inhibition sometimes upregulates total DR4 but does not commonly affect cell surface DR4 level. DR4 is lowly expressed in glioblastoma cells and less important for apoptosis by TRAIL and KPNB1 inhibition. KPNB1 inhibition promotes Noxa expression and Noxa/Mcl-1 interaction, which may free Bak and Bax from Mcl-1 to induce MOMP. However, Noxa upregulation is dispensable for IPZ/TRAIL-induced apoptosis possibly owing to the redundancy and abundance of BH3-only proteins in neutralizing Mcl-1.

KPNB1 inhibition downregulates FLIPs by suppressing cap-dependent translation initiation. One aim of PERK branch activation is to alleviate global protein synthesis by p-eIF2 $\alpha$ to relieve UPR ${ }^{19}$. Besides, changes in mTOR-4EBP1 axis upon UPR also suppress translation ${ }^{36}$. KPNB1 inhibition does not decrease p-mTOR and p-4E-BP1 but increases ATF4-mediated 4E-BP1 expression to inhibit FLIP $_{S}$ translation. KPNB1 inhibition-induced p-eIF2 $\alpha$ upregulates ATF4 but not downstream 4E-BP1, thereby leaving FLIP $_{S}$ expression unaffected. Moreover, ATF4 knockdown does not rescue KPNB1 inhibition/TRAILinduced apoptosis possibly because ATF4 is required for 


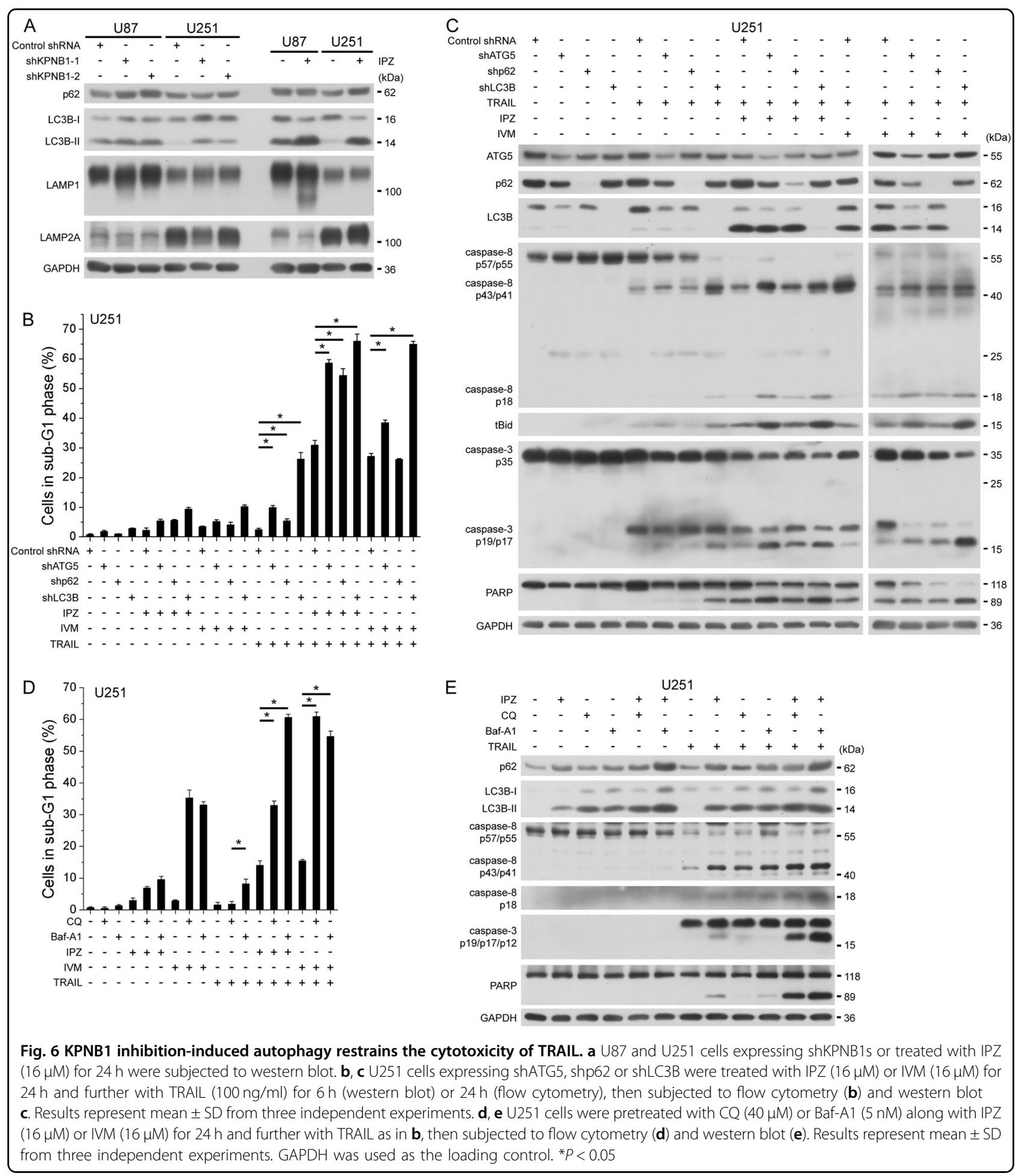

Mcl-1 transcription under UPR ${ }^{37}$. The translation rates of FLIP $_{S}$ and FLIP vary $^{38}$, but those of FLIP ${ }_{S}$ and FLIP ${ }_{L}$ are unknown. Our study showed that within the time both mRNA production and protein degradation are inhibited, FLIP $_{S}$ protein accumulates more than FLIP $_{\mathrm{L}}$ protein. Moreover, FLIP $_{\mathrm{L}}$ translation is irrelevant to $4 \mathrm{E}-\mathrm{BP} 1$ activity but sometimes is controlled by mTOR-S6K1 axis $^{23,39}$. Therefore, translation modes of FLIP Snd FLIP $_{\mathrm{L}}$ differ. We speculate that 4E-BP1 knockdown may unleash FLIP $_{S}$ translation, which competes with FLIP $_{\mathrm{L}}$ translation for resources and further limits FLIP $_{\mathrm{L}}$ expression. We still need to know how $\operatorname{FLIP}_{\mathrm{L}}$ is 

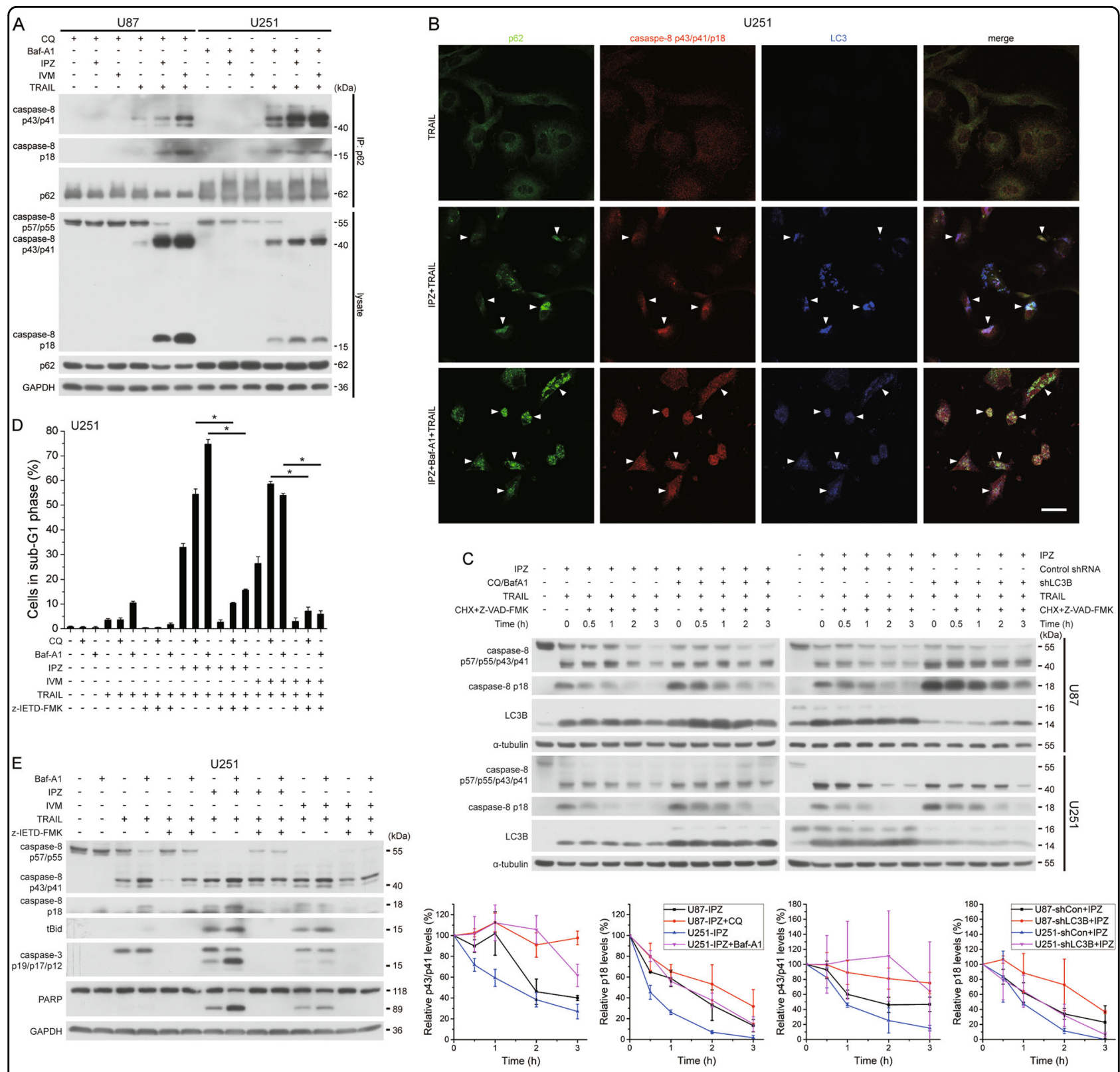

Fig. 7 KPNB1 inhibition-induced autophagy eliminates TRAIL-induced cleaved caspase-8. a U87 and U251 cells were pretreated with CQ $(40 \mu \mathrm{M})$ or Baf-A1 $(5 \mathrm{nM})$ along with IPZ $(16 \mu \mathrm{M})$ or IVM $(16 \mu \mathrm{M})$ for $24 \mathrm{~h}$ and further with TRAlL $(\mathrm{U} 87,50 \mathrm{ng} / \mathrm{ml}$; U251, $100 \mathrm{ng} / \mathrm{ml})$ for $3 \mathrm{~h}$. P62 in cell lysates was immunoprecipitated followed by western blot. b Representative images showed colocalization of p62, caspase-8 p43/p41/p18, and LC3 in U251 cells when treated with Baf-A1 $(5 \mathrm{nM})$ and IPZ $(16 \mu \mathrm{M})$ for $24 \mathrm{~h}$ and further with TRAlL $(100 \mathrm{ng} / \mathrm{ml})$ and z-DEVD-FMK (50 $\mu \mathrm{M})$ for $8 \mathrm{~h}$.

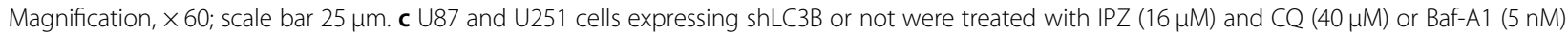
for $24 \mathrm{~h}$ and further with TRAIL (U87, $50 \mathrm{ng} / \mathrm{ml} ; \mathrm{U} 251,100 \mathrm{ng} / \mathrm{ml}$ ) for $2 \mathrm{~h}$. Then, cells were subjected to CHX pulse-chase assay by treating with CHX $(\mathrm{U} 87,20 \mu \mathrm{g} / \mathrm{ml} ; \mathrm{U} 251100 \mu \mathrm{g} / \mathrm{ml})$ and z-VAD-FMK $(20 \mu \mathrm{M})$ for indicated period of time. Representative images of western blot were shown in the upper panel. Quantification of grayscale ratio of cleaved caspase-8/a-tubulin by Photoshop software were shown in the lower panel. Results represent mean \pm SD from two independent experiments. d, e U251 cells were pretreated as in a and further with z-IETD-FMK (20 $\mu$ M) and TRAIL $(100 \mathrm{ng} / \mathrm{ml})$ for $6 \mathrm{~h}$ (western blot) or $24 \mathrm{~h}$ (flow cytometry), then subjected to flow cytometry (d) and western blot (e). Results represent mean \pm SD from three independent experiments. GAPDH and a-tubulin was used as loading controls. ${ }^{*} P<0.05$

downregulated as it is necessary for KPNB1 inhibition/ TRAIL-induced apoptosis.

KPNB1 inhibition-induced autophagy protects glioblastoma cells from proteotoxicity ${ }^{9}$. The role of autophagy in TRAIL receptor signaling is more complicated. Autophagy facilitates incipient TRAIL receptor complex trafficking and caspase- 8 activation ${ }^{40,41}$. However, evidences also support that autophagy negatively regulates 


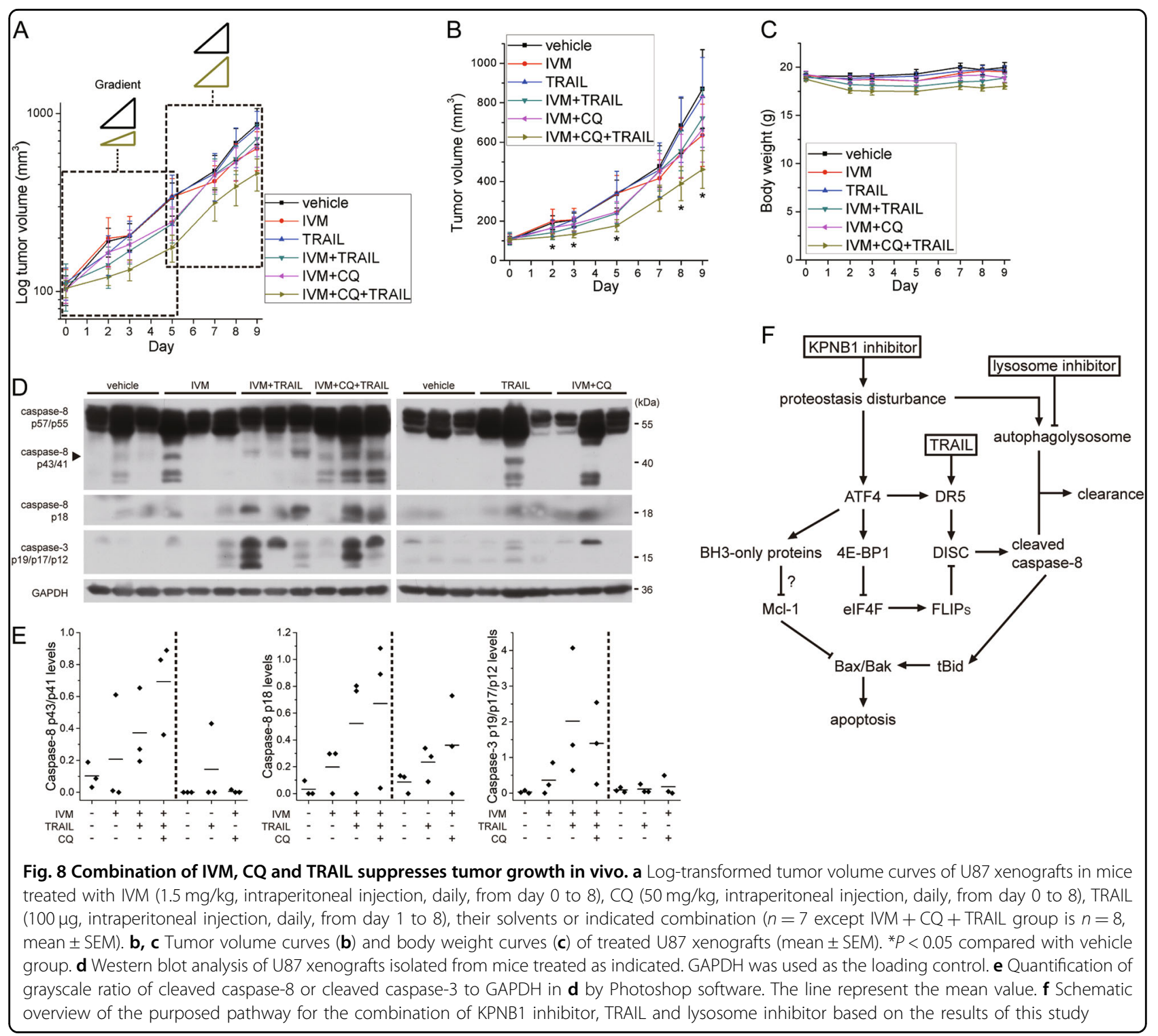

TRAIL-triggered apoptosis. Cells with high autophagic flux and low p62 aggregation are resistant to TRAIL ${ }^{42}$. Autophagosome also impairs membrane localization of death receptors causing TRAIL resistance ${ }^{43}$. Upon TRAIL stimulation, the ubiquitin-proteasome system degrades cleaved caspase- $8^{44}$. However, whether autophagy degrades cleaved caspase- 8 to avoid TRAIL-induced apoptosis is uncertain, though capsase- 8 aggregates colocalize with autophagosomes and lysosomes upon TRAIL stimulation ${ }^{25}$. In this study, genetic inhibition of autophagosome biogenesis or extension or pharmacological inhibition of lysosomal degradation further augments cleaved caspase- 8 level and consequent apoptosis upon KPNB1 inhibitor/TRAIL treatment, which is abrogated by caspase- 8 inhibitor. Blocking lysosomal degradation stabilizes cleaved caspase- 8 with minor effect on procaspase- 8 cleavage. Mechanistically, p62 binds caspase-8 $\mathrm{p} 43 / \mathrm{p} 41$ and p18 following lysosome inhibitor and TRAIL treatment and binds more with KPNB1 inhibitor cotreatment. In concert with previous findings ${ }^{25}$, aggregated cleaved caspase-8, p62, and LC3 colocalized after KPNB1 inhibitor/TRAIL treatment. Thus, p62-labeled cleaved caspase- 8 is targeted by autophagosome for degradation, which disturbs KPNB1 inhibitor/TRAIL-triggered apoptosis. These data challenge the hypothesis that accumulation of p62 aggregates upon autophagy inhibition causes cytotoxicity or facilitates aggregation and cleavage of procaspase- 8 to upregulate cleaved caspase- ${ }^{42}$, as p62 knockdown promoted three drug combination-induced apoptosis. These results underline that autophagy favors initial caspase- 8 activation under TRAIL stimulation or cytotoxic stresses ${ }^{45}$ but impacts more on caspase-8 turnover lowering TRAIL response. 
Overall, our findings provide a potentially novel anticancer mechanism of combining TRAIL, KPNB1 inhibitor and lysosome inhibitor. Combination of KPNB1 inhibitor and TRAIL may be applicable for treating tumors other than glioblastoma in view of data from previous study ${ }^{8}$ and ours (Supplementary Fig. S10A, B) but demands further exploration. Given that TRAIL, IVM and CQ show clinical safety, such combination therapy may rapidly move into anticancer clinical trials.

\section{Materials and methods \\ Cell culture}

Human glioblastoma cell lines U87, U118, U251, and A172 were cultured in DMEM supplemented with 10\% fetal bovine serum, $1 \%$ non-essential amino acid, and $1 \%$ sodium pyruvate (Life technologies, Grand Island, USA). Human fetal astrocytes from cerebral cortex were cultured in astrocyte medium (ScienCell Research Labortories, Carlsbad, USA) with $2 \%$ fetal bovine serum. U87 and U251 were purchased in April 7, 2017 (purchase order, 85676) and U118 and A172 were purchased in July 13, 2018 (purchase order, 115354) from cell bank of Chinese Academy of Sciences (Shanghai, China), where they were authenticated by means of STR profiling. Human astrocytes were obtained from ScienCell Research Laboratories. All cells were maintained under standard cell culture conditions at $37^{\circ} \mathrm{C}$ and $5 \% \mathrm{CO}_{2}$.

\section{Antibodies and reagents}

Primary antibodies used in this study were listed below: antibodies against $\alpha$-tubulin (HRP-conjugated) (HRP66031), KPNB1 (10077-1-AP) (ProteinTech Group, Wuhan, China), LAMP2A (ab125068) (Abcam, Canbridge, UK), 4E-BP1 (9644), ATF4 (11815), ATG5 (12994), Bax (5023), Bak (12105), Bid (2002), caspase-3 (9662), caspase-8 (9746) (for western blot and immunoprecipitation), CHOP (2895), cleaved caspase-8 (9496), DR4 (42533), DR5 (8074), eIF4E (2067), eIF4G (2469), FLIP (56343), LAMP1 (9091), LC3A/B (Alexa Fluor 647) (13394), LC3B (3868), Mcl-1 (5453), MeCP2 (3456), mouse mAb IgG1 isotype control (5415) (for functional neutralization), Noxa (14766), p-4E-BP1 (T37/46) (2855), p62 (8025), p62 (Alexa Fluor 488) (8833), PARP (9532), Puma (12450) (Cell Signaling Technology, Beverly, USA), caspase-8 (sc-6136) (for detection of caspase-8 in caspase8 immunoprecipitant) (Santa Cruz Biotechnology, Santa Cruz, USA), c-Cbl (610441), FADD (556402), Mcl-1 (for immunoprecipitation) (559027) (BD Biosciences, San Jose, USA), ATF3 (BS2261), DR4 (BS2238), eIF2 $\alpha$ (BS3651), p-eIF2 $\alpha$ (S51) (BS4787) (Bioworld Technology, Nanjing, China), Lamin B (BA1228) (Boster, Wuhan, China), GAPDH (KC-5G5) (Kangchen, Shanghai, China), APC anti-human CD261 (DR4, TRAIL-R1) (307208), APC anti-human CD262 (DR5, TRAIL-R2) (307408),
APC mouse IgG1, $\kappa$ isotype ctrl antibody (400119) (all for flow cytometry) (BioLegend, San Diego, USA), antiTRAIL-R1 (human), mAb (HS101) (AG-20B-0022PF) and anti-TRAIL-R2 (human), mAb (HS201) (AG-20B0023PF) (both for functional neutralization) (AdipoGen AG, Liestal, Switzerland). Anti-mouse (7076) or antirabbit (7074) secondary antibodies (horseradish peroxidase-conjugated) were acquired from Cell Signaling Technology. Alexa Fluor 546 goat anti-rabbit antibody (A-11010) was from Life Technologies (Carlsbad, USA).

Reagents and kits used in this study were listed below: importazole (IPZ) (Merck Millipore, Darmstadt, Germany), PMSF, propidium iodide (Sigma-Aldrich, St. Louis, USA), CalPhos Mammalian Transfection Kit (TaKaRa Bio, Kusatsu, Japan), protease inhibitor cocktail, RNase A, Click-iT ${ }^{\mathrm{mm}}$ Plus OPP Protein Synthesis Assay Kit Alexa Fluor $^{\text {ri }} 488$ picolyl azide (Thermo Scientific, Waltham, USA), phosphatase inhibitor, thiazolyl blue tetrazolium bromide (Sangon, Shanghai, China), cell lysis buffer for Western and IP, cycloheximide (CHX), nuclear and cytoplasmic protein extraction kit, RIPA (Beyotime, Nantong, China), LightCycler 480 SYBR Green I Master, protein A agarose, protein $\mathrm{G}$ agarose (Roche Diagnositics, Indianapolis, USA), bafilomycin A1 (Baf-A1), ivermectin (IVM), MG132, z-VAD-FMK (Selleck, Shanghai, China), Act $\mathrm{D}$, chloroquine diphosphate (CQ), z-DEVD-FMK, zIETD-FMK (Medchem Express, Monmouth Junction, USA), human recombinant TRAIL (PeproTech, Rocky Hill, USA), iScript Reverse Transcription Supermix (BioRad, Berkeley, USA), immobilized 2' $2^{\prime}-$ EDA- $\mathrm{m}^{7}$ GTP (Jena Bioscience, Jena,Germany) were used in this study.

\section{Lentivirus-mediated gene transduction}

Short hairpin RNAs (shRNAs) targeting human KPNB1, DR5, ATF4, CHOP, Noxa, 4E-BP1, ATG5, p62, LC3B, and a scrambled (control) shRNA were inserted into the lentiviral vector pLKD-CMV-GFP-U6-shRNA. Coding DNAs of human c-Cbl, non-degradable Mcl-1 (T92A) mutant, FLIP $_{\mathrm{L}}$, FLIP and eIF2 $\alpha$ (S52A) were inserted into pCDH-CMV-MCS-EF1-copGFP. Lentiviral plasmids, gag/pol packaging vector and VSVG encoding plasmid were transfected into 293T cells using CalPhos Mammalian Transfection Kit according to the manufacturer's protocol. Culture medium was harvested $48 \mathrm{~h}$ and $72 \mathrm{~h}$ after transfection and ultracentrifugated to obtain hightiter purified preparation. The sequences of shRNAs used were listed in the Supplementary Data.

\section{Western blot}

After collection, cells were lysed in RIPA supplemented with PMSF, phosphatase inhibitor, and protease inhibitor cocktail. Western blot was carried out as previously described $^{46}$. Grayscale of protein bands was analyzed by Photoshop CS4 software. 


\section{MTT assay}

Cell viability was measured by MTT assay performed as previously described $^{46}$.

\section{Flow cytometry assay}

To measure cell surface DR4 and DR5 expression, intact cells were stained with APC anti-human DR4 or DR5 antibody or IgG isotype ctrl antibody for $30 \mathrm{~min}$ at $4{ }^{\circ} \mathrm{C}$. To measure apoptosis, fixed cells were stained with propidium iodide as previously described ${ }^{46}$. After staining, cells were analyzed using a BD LSR II flow cytometer. Sub-diploid cells were considered apoptotic. The mean fluorescence intensity and proportion of sub-dipliod cells were analyzed by FlowJo 7.6.1 software.

\section{Immunoprecipitation and DISC analysis}

Cells were lysed on ice in cell lysis buffer for Western and IP (20 mM Tris (pH 7.5), $150 \mathrm{mM} \mathrm{NaCl}, 1 \%$ Triton X$100)$. Lysates were adjusted to have equal protein concentrations and incubated with indicated antibodies overnight, and with additional protein A agarose or protein $\mathrm{G}$ agarose at $4{ }^{\circ} \mathrm{C}$ for $3 \mathrm{~h}$. For DISC analysis, cells were treated with $20 \mu \mathrm{M} z$-VAD-FMK for $5 \mathrm{~h}$ and further with or without $100 \mathrm{ng} / \mathrm{ml}$ TRAIL for $1 \mathrm{~h}$. Cells were lysed in lysis buffer (50 mM Tris- $\mathrm{HCl}$ (pH 7.5), $150 \mathrm{mM} \mathrm{NaCl}, 1 \%$ Triton X-100, 1 mM EDTA, 10\% glycerol). Lysates were incubated with caspase-8 (9746) antibody overnight to immunoprecipitate DISC. Precipitates were washed three times with lysis buffer before adding SDS-PAGE loading buffer and denaturation. Precipitates and lysates were then subjected to western blot.

\section{RNA extraction and real-time PCR}

RNA was extracted, reverse transcribed and analyzed by quantitative real-time PCR. Relative gene mRNA levels were normalized to that of GAPDH. The comparative $\mathrm{Ct}$ method was used to calculate fold changes in expression. Primer sets for PCR were obtained from PrimerBank are listed in the Supplementary Data.

\section{Cap-binding assay}

Cell lysates were incubated with $\mathrm{m}^{7} \mathrm{GTP}$ agarose at $4{ }^{\circ} \mathrm{C}$ for $3 \mathrm{~h}$ to capture eIF4E and its binding proteins. Following procedures were performed in the same procedure as immunoprecipitation.

\section{Protein synthesis assay}

Cells were plated in 96-well culture plates. After treatment with indicated drugs, cells were incubated with $20 \mu \mathrm{M}$ O-propargyl-puromycin (OPP) for $0.5 \mathrm{~h}$. OPP detection and normalization to cell number were performed according to the manufacturer's protocol. FITC intensity was measured by multi-mode microplate reader.

\section{Immunofluorescence}

Cells cultured onto coverslips were fixed with $4 \%$ paraformaldehyde at room temperature for $15 \mathrm{~min}$. Cells were incubated with blocking buffer (5\% bovine serum albumin and $0.1 \%$ Triton in PBS) for $1 \mathrm{~h}$ and stained with antibodies and Hoechst 33342 at $4{ }^{\circ} \mathrm{C}$ overnight. Fluorescent signals were observed with a Nikon FN1 confocal microscope at $\times 60$ magnification.

\section{Expression and purification of recombinant TRAIL}

The cDNA sequence encoding the extracellular portion of human TRAIL (aa 114-281) was inserted into pGEX6p-1. Recombinant GST-TRAIL was expressed in Rosetta bacteria as the expression host. The overnight seed culture was diluted 500-fold into LB broth and incubated at $37^{\circ} \mathrm{C}$. When $\mathrm{OD}_{600}$ of the broth is around $0.6-1$, isopropyl-1-thio- $\beta$-D-galactopyranoside $(0.5 \mathrm{mM})$ was added to induce recombinant protein expression, and the culture was incubated at overnight $25^{\circ} \mathrm{C}$. Bacteria were harvested and resuspended in PBS $+0.5 \%$ Tween $20+$ proteasome inhibitor and then cracked by high pressure homogenizer. Recombinant GST-TRAIL was isolated from the soluble fraction by affinity chromatography using GlutathioneSepharose $4 B$ and soluble TRAIL was seperated by prescission protease. The purity of recombinant TRAIL was confirmed by SDS-PAGE and Coomassie Blue staining. The oligomeric state was analyzed by gel filtration chromatography. The protein concentration was calculated by comparing the grayscale of its band with that of BSA standard.

\section{In vivo study}

Female BALB/c nude mice (5 weeks, Shanghai Lingchang Bioscience Company, China) were maintained in the pathogen-free environment. All experimental procedures were approved by the Institutional Animal Care and Use Committee of the Institute of Neuroscience, Chinese Academy of Sciences. U87 cells $\left(5 \times 10^{6}\right)$ in $150 \mu$ l serumfree DMEM were inoculated subcutaneously into the area under the left flank of mice. When average tumor volume reached approximately $100 \mathrm{~mm}^{3}$, mice were randomized into six groups $(n=7$ except IVM + CQ + TRAIL group is $n=8)$ : vehicle, IVM, TRAIL, IVM + TRAIL, IVM + CQ, IVM + CQ + TRAIL. Mice were pretreated with IVM $(6 \mathrm{mg} / \mathrm{kg}$, in $1.5 \% \mathrm{DMSO}+58.5 \%$ 1, 2-propylene glycol $+40 \%$ water $)$, CQ $(50 \mathrm{mg} / \mathrm{kg}$, in saline) or solvents by intraperitoneal injection. One day later, mice were treated with IVM, CQ, TRAIL $(100 \mu \mathrm{g}$, in PBS $+2 \%$ Tween 20) or solvents by intraperitoneal injection daily for 8 days. Tumor length and width were measured by caliper. Tumor volume was calculated using the formula: $\mathrm{V}=0.5 \times$ length $\times$ width $^{2}$. One day after the final treatment, mice were euthanized and tumors were immediately isolated and lysed for western blot. 


\section{Statistical analysis}

OriginPro 8 software (OriginLab Corporation, Northampton, USA) was used for data analysis and graphing. Results are expressed as mean \pm SD unless indicated. The two-tailed unpaired $t$-test was used to determine significant differences between the mean values of groups, with statistical significance defined as $p<0.05$.

\section{Acknowledgements}

This work was supported by the National Natural Science Foundation of China [grant numbers 31490591]; Ministry of Science and Technology [grant number 2016YFA0501002].

\section{Author details \\ ${ }^{1}$ State Key Laboratory of Neuroscience, Institute of Neuroscience, CAS Center for Excellence in Brain Science and Intelligence Technology, Chinese Academy of Sciences, Shanghai, China. ${ }^{2}$ Shanghai Mental Health Center, Shanghai Jiao Tong University School of Medicine, Shanghai, China. ${ }^{3}$ University of Chinese Academy of Sciences, Beijing, China. ${ }^{4}$ School of Life Science and Technology, ShanghaiTech University, Shanghai, China}

\section{Author contributions}

Z-Q.X. directed the overall project. Z-Q.X. and Z-C.Z. designed the study. Z-C.Z., J-W.L., C.Y., M-J.L and R-J.W. performed the experiments. Z-C.Z. analyzed the data and wrote the main manuscript. All authors reviewed the manuscript.

\section{Conflict of interest}

The authors declare that they have no conflict of interest.

\section{Publisher's note}

Springer Nature remains neutral with regard to jurisdictional claims in published maps and institutional affiliations.

Supplementary Information accompanies this paper at (https://doi.org/ 10.1038/s41419-019-1383-x).

Received: 29 August 2018 Revised: 16 December 2018 Accepted: 9 January 2019

Published online: 11 February 2019

\section{References}

1. Yang, A., Wilson, N. S. \& Ashkenazi, A. Proapoptotic DR4 and DR5 signaling in cancer cells: toward clinical translation. Curr. Opin. Cell Biol. 22, 837-844 (2010).

2. Lemke, J., von Karstedt, S., Zinngrebe, J. \& Walczak, H. Getting TRAll back on track for cancer therapy. Cell Death Differ. 21, 1350-1364 (2014).

3. den Hollander, M. W. et al. Translating TRAlL-receptor targeting agents to the clinic. Cancer Lett. 332, 194-201 (2013).

4. Kuijlen, J. M., Bremer, E., Mooij, J. J., den Dunnen, W. F. \& Helfrich, W. Review: on TRAIL for malignant glioma therapy? Neuropathol. Appl. Neurobiol. 36, 168-182 (2010).

5. Giri, D. K. et al. Endosomal transport of ErbB-2: mechanism for nuclear entry of the cell surface receptor. Mol. Cell. Biol. 25, 11005-11018 (2005).

6. Liang, P. et al. KPNB1, XPO7 and IPO8 mediate the translocation ofNF-kappaB/ p65 into the nucleus. Traffic 14, 1132-1143 (2013).

7. Cimica, V., Chen, H. C., Iyer, J. K. \& Reich, N. C. Dynamics of the STAT3 transcription factor: nuclear import dependent on Ran and importin-beta1. PLoS ONE 6, e20188 (2011).

8. Kojima, Y. et al. Importin beta1 protein-mediated nuclear localization of death receptor 5 (DR5) limits DR5/tumor necrosis factor (TNF)-related apoptosisinducing ligand (TRAIL)-induced cell death of human tumor cells. J. Biol. Chem. 286, 43383-43393 (2011).

9. Zhu Z. C., Liu J. W., Li K., Zheng J., Xiong Z. Q. KPNB1 inhibition disrupts proteostasis and triggers unfolded protein response-mediated apoptosis in glioblastoma cells. Oncogene. 37, 2936-2952 (2018).
10. Martin-Perez, R., Niwa, M. \& Lopez-Rivas, A. ER stress sensitizes cells to TRAlL through down-regulation of FLIP and Mcl-1 and PERK-dependent up-regulation of TRAIL-R2. Apoptosis 17, 349-363 (2012).

11. Teng, Y. et al. Inhibition of elF2alpha dephosphorylation enhances TRAILinduced apoptosis in hepatoma cells. Cell Death Dis. 5, e1060 (2014).

12. van Roosmalen, I. A. M. et al. The ER stress inducer DMC enhances TRAlLinduced apoptosis in glioblastoma. + 3, 495 (2014).

13. Laguinge, L. M. et al. DR5 receptor mediates anoikis in human colorectal carcinoma cell lines. Cancer Res. 68, 909-917 (2008).

14. Chen, J. J., Chou, C. W., Chang, Y. F. \& Chen, C. C. Proteasome inhibitors enhance TRAlL-induced apoptosis through the intronic regulation of DR5: involvement of NF-kappa B and reactive oxygen species-mediated p53 activation. J. Immunol. 180, 8030-8039 (2008).

15. Son, Y. G. et al. Silibinin sensitizes human glioma cells to TRAlL-mediated apoptosis via DR5 up-regulation and down-regulation of c-FLIP and survivin. Cancer Res. 67, 8274-8284 (2007).

16. Yoon, M. J. et al. Monensin, a polyether ionophore antibiotic, overcomes TRAlL resistance in glioma cells via endoplasmic reticulum stress, DR5 upregulation and C-FLIP downregulation. Carcinogenesis 34, 1918-1928 (2013).

17. Song, J. J. et al. c-Cbl-mediated degradation of TRAIL receptors is responsible for the development of the early phase of TRAIL resistance. Cell. Signal. 22, 553-563 (2010).

18. Rodriguez, D., Rojas-Rivera, D. \& Hetz, C. Integrating stress signals at the endoplasmic reticulum: The BCL-2 protein family rheostat. Biochim. Biophys. Acta 1813, 564-574 (2011).

19. Sano, R. \& Reed, J. C. ER stress-induced cell death mechanisms. Biochim. Biophys. Acta 1833, 3460-3470 (2013).

20. Van Der Kelen, K., Beyaert, R., Inze, D. \& De Veylder, L. Translational control of eukaryotic gene expression. Crit. Rev. Biochem. Mol. Biol. 44, 143-168 (2009).

21. Yamaguchi, S. et al. ATF4-mediated induction of 4E-BP1 contributes to pancreatic beta cell survival under endoplasmic reticulum stress. Cell. Metab. 7 269-276 (2008).

22. Tominaga, R. et al. The JNK pathway modulates expression and phosphorylation of 4E-BP1 in MIN6 pancreatic beta-cells under oxidative stress conditions. Cell. Biochem. Funct. 28, 387-393 (2010).

23. Panner, A., James, C. D., Berger, M. S. \& Pieper, R. O. mTOR controls FLIPS translation and TRAIL sensitivity in glioblastoma multiforme cells. Mol. Cell. Biol. 25, 8809-8823 (2005)

24. Jin, Z. et al. Cullin3-based polyubiquitination and p62-dependent aggregation of caspase-8 mediate extrinsic apoptosis signaling. Cell 137, 721-735 (2009).

25. Hou, W., Han, J., Lu, C., Goldstein, L. A. \& Rabinowich, H. Autophagic degradation of active caspase-8: a crosstalk mechanism between autophagy and apoptosis. Autophagy 6, 891-900 (2010).

26. Ganten, T. M. et al. Preclinical differentiation between apparently safe and potentially hepatotoxic applications of TRAIL either alone or in combination with chemotherapeutic drugs. Clin. Cancer Res. 12, 2640-2646 (2006).

27. Liu, H. et al. Improvement of Pharmacokinetic Profile of TRAIL via Trimer-Tag Enhances its Antitumor Activity in vivo. Sci. Rep. 7, 8953 (2017).

28. Siegelin, M. D. Utilization of the cellular stress response to sensitize cancer cells to TRAlL-mediated apoptosis. Expert. Opin. Ther. Targets 16, 801-817 (2012).

29. Iurlaro R., et al. Glucose Deprivation Induces ATF4-Mediated Apoptosis through TRAIL Death Receptors. Mol Cell Biol. 37, pii: e00479-16 (2017).

30. Liu, H. et al. 2-Deoxy-D-glucose enhances TRAlL-induced apoptosis in human melanoma cells through XBP-1-mediated up-regulation of TRAIL-R2. Mol. Cancer 8, 122 (2009).

31. Dufour, F. et al. TRAlL receptor gene editing unveils TRAlL-R1 as a master player of apoptosis induced by TRAIL and ER stress. Oncotarget 8, 9974-9985 (2017).

32. Jung, K. J., Min, K. J., Bae, J. H. \& Kwon, T. K. Carnosic acid sensitized TRAlLmediated apoptosis through down-regulation of c-FLIP and BCl-2 expression at the post translational levels and CHOP-dependent up-regulation of DR5, Bim, and PUMA expression in human carcinoma caki cells. Oncotarget 6, 1556-1568 (2015)

33. Liu, X. et al. Afatinib down-regulates MCL-1 expression through the PERKelF2alpha-ATF4 axis and leads to apoptosis in head and neck squamous cell carcinoma. Am. J. Cancer Res. 6, 1708-1719 (2016).

34. Fritsch, R. M., Schneider, G., Saur, D., Scheibel, M. \& Schmid, R. M. Translational repression of MCL-1 couples stress-induced elF2 alpha phosphorylation to mitochondrial apoptosis initiation. J. Biol. Chem. 282, 22551-22562 (2007).

35. Gomez-Bougie, P., Halliez, M., Moreau, P., Pellat-Deceunynck, C. \& Amiot, M. Repression of $\mathrm{Mcl}-1$ and disruption of the Mcl-1/Bak interaction in myeloma 
cells couple ER stress to mitochondrial apoptosis. Cancer Lett. 383, 204-211 (2016).

36. Preston, A. M. \& Hendershot, L. M. Examination of a second node of translational control in the unfolded protein response. J. Cell. Sci. 126(Pt 18), 4253-4261 (2013).

37. $\mathrm{Hu}$, J. et al. Activation of ATF4 mediates unwanted Mcl-1 accumulation by proteasome inhibition. Blood 119, 826-837 (2012).

38. Ueffing, N. et al. A single nucleotide polymorphism determines protein isoform production of the human c-FLIP protein. Blood 114, 572-579 (2009).

39. Zhang, T., Wang, X., He, D., Jin, X. \& Guo, P. Metformin sensitizes human bladder cancer cells to TRAlL-induced apoptosis through mTOR/S6K1-mediated downregulation of c-FLIP. Anticancer Drugs 25, 887-897 (2014).

40. Horova, V. et al. Inhibition of vacuolar ATPase attenuates the TRAll-induced activation of caspase- 8 and modulates the trafficking of TRAlL receptosomes. Febs. J. 280, 3436-3450 (2013).
41. Young, M. M. et al. Autophagosomal membrane serves as platform for intracellular death-inducing signaling complex (iDISC)-mediated caspase-8 activation and apoptosis. J. Biol. Chem. 287, 12455-12468 (2012).

42. Singh, $\mathrm{K}$. et al. Autophagic flux determines cell death and survival in response to Apo2L/TRAIL (dulanermin). Mol. Cancer 13, 70 (2014).

43. $\mathrm{Di}, \mathrm{X}$. et al. Accumulation of autophagosomes in breast cancer cells induces TRAIL resistance through downregulation of surface expression of death receptors 4 and 5. Oncotarget 4, 1349-1364 (2013).

44. Gonzalvez, F. et al. TRAF2 Sets a threshold for extrinsic apoptosis by tagging caspase-8 with a ubiquitin shutoff timer. Mol. Cell 48, 888-899 (2012).

45. Huang, S., Okamoto, K., Yu, C. \& Sinicrope, F. A. p62/sequestosome-1 upregulation promotes ABT-263-induced caspase-8 aggregation/activation on the autophagosome. J. Biol. Chem. 288, 33654-33666 (2013).

46. Zhu, Z. et al. ZFX regulates glioma cell proliferation and survival in vitro and in vivo. J. Neurooncol. 112, 17-25 (2013). 